\title{
Cuidado interprofissional em saúde mental via teleatendimento em farmácia universitária frente à pandemia da COVID-19
}

\section{Interprofessional mental health care via call center at a university pharmacy in the face of the covid-19 pandemic}

\author{
Viviane Alvino da Guia ${ }^{*}$, Alicia Maria Rocha do Amaral ${ }^{1}$, Luan Diniz Pessoa ${ }^{1}$, Camila Gurgel \\ de Paula ${ }^{1}$, Thais Teles de Souza ${ }^{1}$, Ernani Vieira de Vasconcelos Filho ${ }^{2}$, Cintia Caldas Rios ${ }^{3 .}$ \\ Walleri Christini Torelli Reis ${ }^{1}$
}

\begin{abstract}
RESUMO
A pandemia da COVID-19 demandou adaptações de inúmeros serviços, diante disso o presente trabalho busca relatar a adaptação de um núcleo de atendimento interprofissional em saúde mental localizado em uma farmácia universitária frente à pandemia. Para tanto se analisou dados de pacientes atendidos no período inicial da pandemia. Analisou-se perfil epidemiológico, farmacoterapêutico, metodologia utilizada para o atendimento e tipos de intervenções. Foram analisados 81 pacientes, $67 \%$ do sexo feminino, faixa etária prevalente 18 a 29 anos. Sobre os teleatendimentos e telemonitoramentos, $93 \%$ foram realizados pelo farmacêutico gestor do caso e/ou extensionistas. Em relação às consultas interprofissionais, $68 \%$ foram relacionadas a sugestão de renovação de prescrição médica. Nesse sentido, torna-se evidente a relevância do serviço interprofissional na saúde mental, bem como a atuação do Farmacêutico e sua adaptação em momentos de urgências sanitárias.
\end{abstract}

Palavras-chave: Teleatendimento em saúde; Saúde mental; Cuidado interprofissional; Cuidado FarmacÊtico; COVID-19.

\section{ABSTRACT}

The COVID-19 pandemic required adaptations from numerous services, and as a result, this paper seeks to report the adaptation of an interprofessional mental health care center located in a university pharmacy in the face of the pandemic. For this purpose, data from patients assisted in the initial period of the pandemic were analyzed. The epidemiological profile, pharmacotherapeutic, methodology used for care and types of interventions were analyzed. $\mathrm{N}=81$ patients, $67 \%$ female, prevalent age range 18 to 29 years. About teleservices and telemonitoring, $93 \%$ were carried out by the case manager pharmacist and/or extension workers. Regarding interprofessional consultations, $68 \%$ related to the suggestion of medical prescription renewal. In this sense, the relevance of the interprofessional service in mental health becomes evident, as well as the role of the Pharmacist and his adaptation in times of health emergencies.

Keywords: Health call center; Mental health; Interprofessional care; Pharmaceutical care; COVID-19.

\footnotetext{
1 Departamento de Ciências Farmacêuticas, Centro de Ciências da Saúde, Universidade Federal da Paraíba.*E-mail: vivianealvino@live.com

2 Departamento de Promoção da Saúde, Centro de Ciências Médicas, Universidade Federal da Paraíba.

${ }^{3}$ Universidade Federal de Sergipe.
} 
INTRODUÇÃOO

A situação sanitária instalada no mundo devido à pandemia causada pelo vírus SARS-CoV-2, denominado como novo Coronavírus, ocasionou a constante necessidade de adequação dos indivíduos em suas diversas atividades cotidianas a fim de conter a disseminação do vírus, fato esse que pôde ser observado nas medidas de distanciamento social (AFONSO, 2020). Diante de uma situação emergencial como a vivenciada atualmente, é notório que a saúde mental da população de modo em geral e principalmente de profissionais que atuam na linha de frente esteja comprometida(ORNELL et al. 2020).

Os transtornos mentais são considerados um problema de saúde pública, com impacto direto na qualidade de vida e capacidade laboral dos indivíduos. O Brasil é considerado um dos mais acometidos a nível mundial (OMS, 2017). A pandemia de 2019, visto o isolamento social, medo de adoecimento e vivência de perdas, apresentou impacto direto no adoecimento e agravamento de transtornos mentais, tanto no contexto dos profissionais de saúde como na comunidade (PEREIRA et al. 2020).

Vale ressaltar que os transtornos mentais, visto sua complexidade e necessidade de manter a autonomia do paciente, necessitam de acompanhamento periódico com finalidade de educação em saúde, avaliação do estado clínico e eventuais alterações na farmacoterapia (BEZERRA et al. 2016; VASCONCELOS et al. 2016).

Esses cuidados, por sua vez, vão além de consultas médicas e envolvem um cuidado interprofissional. Essa abordagem é importante a fim de estimular aceitação do paciente, adesão terapêutica e melhora em desfechos em saúde. Atualmente existe uma ampla diversidade na equipe capacitada para atuar na área da saúde mental, podendo ser ela composta por médico, farmacêutico, assistente social, psicólogo, terapeuta ocupacional, enfermeiro, dentre outros(SEVERO; DIMENSTEIN, 2011; BERTAZONE et al. 2016; NDIBU MUNTU KEBA KEBE et al. 2019b).

Devido a fundamental importância na utilização de terapia medicamentosa no manejo de transtornos mentais, a presença do profissional farmacêutico é indiscutível, visto que ele possui atribuições que vão desde educação em saúde e dispensação de medicamentos até a revisão da farmacoterapia e acompanhamento farmacoterapêutico, conduzindo avaliação de risco-benefício da farmacoterapia, considerando os desfechos 
do paciente e intervindo junto ao paciente, família e comunidade condizendo com as Resoluções 585 e 586 de 2013 do Conselho Federal de Farmácia, a Portaria do Ministério da Saúde n 344/98 e com a Lei 13.021/2014. Desse modo, o farmacêutico é um profissional apto para atuar na promoção, proteção e recuperação da saúde em suas diversas vertentes, dentre elas a saúde mental (BRASIL 2013; ZANELLA;AGUIAR.STORPIRTIS, 2015; SILVA; LIMA, 2017).

Considerando a situação epidemiológica atual, o cuidado interprofissional voltado para a saúde mental em períodos de surtos de doenças infecto-contagiosas é essencial, tanto em relação à novas demandas como para aqueles que já necessitavam de cuidado anteriormente. Em ambos os casos a saúde mental estará gravemente afetada devido as incertezas, desestabilidade financeira, solidão e de modo geral pelo medo do desconhecido(AFONSO; FIGUEIRA, 2020; ORNELL et al. 2020).

Assim, se faz necessário a execução de estratégias que auxiliem nesse momento de urgência sanitária, favorecendo tanto o manejo do cuidado da saúde mental, bem como das demais doenças de caráter crônico. Esses casos necessitam de flexibilidades que possibilitem um suporte, de maneira a contribuir com a contenção máxima da disseminação do agente infecto- contagioso, como nos casos de utilização de teleatendimentos, que já são amplamente utilizados em outros países(USHER; BHULLAR; JACKSON, 2020; VELASQUEZ, 2020).

No Brasil, a metodologia de telemedicina não era normatizada, porém em virtude da instalação da pandemia houve modificações na legislação, passando a ser permitida em caráter emergencial, de acordo com a Lei № 13989 de 15/04/2020. Inclusive a telemedicina passou a ser uma das ferramentas para monitorização da COVID-19 em todo o território brasileiro(HARZHEIM et al. 2020).

Portanto, torna-se evidente a importância da continuidade da prestação de serviço em saúde mental para a comunidade diante da situação de infortúnio causada pela pandemia da COVID- 19. Isto posto, o objetivo deste trabalho é caracterizar as atividades interprofissionais voltadas para o âmbito da saúde mental realizadas via teleatendimento em uma Farmácia Universitária no início dessa urgência sanitária. 


\section{MÉTODOS}

\section{Delineamento do estudo}

Foi realizada uma pesquisa observacional com delineamento transversal. Os dados coletados foram referentes às consultas farmacêuticas e/ou interprofissionais realizadas entre os meses de abril e junho de 2020, período inicial de adaptação do serviço para o modelo de teleatendimento.

\section{Critérios de inclusão}

Indivíduos submetidos a atendimentos virtuais voltados a cuidados em saúde mental durante o período de distanciamento social causado pela pandemia da COVID19. Foram incluídos os dados obtidos de todos os prontuários dos pacientes atendidos durante o período determinado previamente, sejam estes prontuários eletrônicos ou físicos.

\section{Critérios de inclusão}

Não ter sido atendido no período que compreende ao início da pandemia da COVID-19.

\section{As variáveis estudadas}

Para definição do perfil epidemiológico foram o sexo, a faixa etária e as comorbidades dos pacientes. Para o perfil de utilização de medicamentos, quantificou-se o número de medicamentos que usa, bem como as substâncias e classes de medicamentos.

\section{Aspectos éticos}

O trabalho contou com aprovação de conselho de ética da Universidade a qual a farmácia escola está inserida, através do CAAE 97906118.3.0000.5188, para análise de dados referentes aos serviços realizados pelo ambulatório.

\section{Caracterização do tipo de intervenção}

Para tanto houve inicialmente uma distinção entre os teleatendimentos, se foram teleconsultas ou telemonitoramento. Após isso, foi feita uma discriminação dos tipos de 
intervenções que foram realizadas, como exemplo: sugestão de diminuição ou aumento de dose; sugestão de adição ou remoção de medicamentos na farmacoterapia; renovação de prescrição; encaminhamento para outra especialidade; entre outros.

\section{Instrumento para coleta de dados}

A obtenção dos dados se deu por meio de informações contidas nos prontuários (físicos ou digitais) utilizando um instrumento para coleta de dados elaborado através de um formulário virtual (Google Forms).

\section{Análises estatísticas}

Os dados foram organizados em planilhas do excel obtidas automaticamente do preenchimento do formulário virtual. A partir dessas, foram originados gráficos descritivos, com o intuito de apurar dados primordiais referentes à pesquisa.

\section{RESULTADOS}

Perfil demográfico dos pacientes vinculados ao núcleo interprofissional de saúde mental

Os pacientes que tiveram dados dos prontuários utilizados para o desenvolvimento desse estudo são os que receberam atendimento no período que compreende o início da pandemia da COVID-19, correspondendo ao período de adaptação do atendimento que já acontecia no núcleo interprofissional de saúde mental em questão.

Foram incluídos 81 indivíduos na análise de dados, entre estes houve uma maior prevalência do sexo feminino com aproximadamente $66 \%$ dos prontuários utilizados, enquanto cerca de $33 \%$ correspondiam a indivíduos do sexo masculino.

A faixa etária variou de 16 a 75 anos de idade, sendo as idades de 18 a 29 anos as mais prevalentes entre os prontuários investigados. A tabela 1 informa a distribuição quanto ao sexo e idade dos pacientes vinculados ao núcleo interprofissional durante o período de interesse para o estudo:

Tabela 1 - Distribuição de dados da população atendida no núcleo de atendimento interprofissional de saúde mental no período inicial da pandemia da COVID-19, quanto ao sexo e faixa etária 


\begin{tabular}{cccc} 
Dados pessoais & Variáveis & Frequência & Porcentagem \\
\hline \multirow{2}{*}{ Sexo } & Fem. & 54 & $67 \%$ \\
& Masc. & 27 & $33 \%$ \\
\hline \multirow{2}{*}{ Idade } & $<16$ anos & 1 & $1 \%$ \\
& $18-29$ anos & 32 & $43 \%$ \\
& $30-45$ anos & 20 & $27 \%$ \\
& $49-59$ anos & 13 & $18 \%$ \\
& $>60$ anos & 4 & $5 \%$ \\
\hline
\end{tabular}

Fonte: Dados da pesquisa

\section{Perfil nosológico}

No que se refere ao diagnóstico dos pacientes, notou-se que maior parte dos indivíduos possuía diagnóstico de transtorno ansioso, representando $76 \%$ dos prontuários analisados (Gráfico 1), seguido do transtorno depressivo que compreendeu ao diagnóstico presente em $54 \%$ dos prontuários verificados e o transtorno afetivo bipolar (TAB) identificado em $13 \%$ dos prontuários.

Gráfico 1 - Transtornos mentais verificados nos prontuários analisados

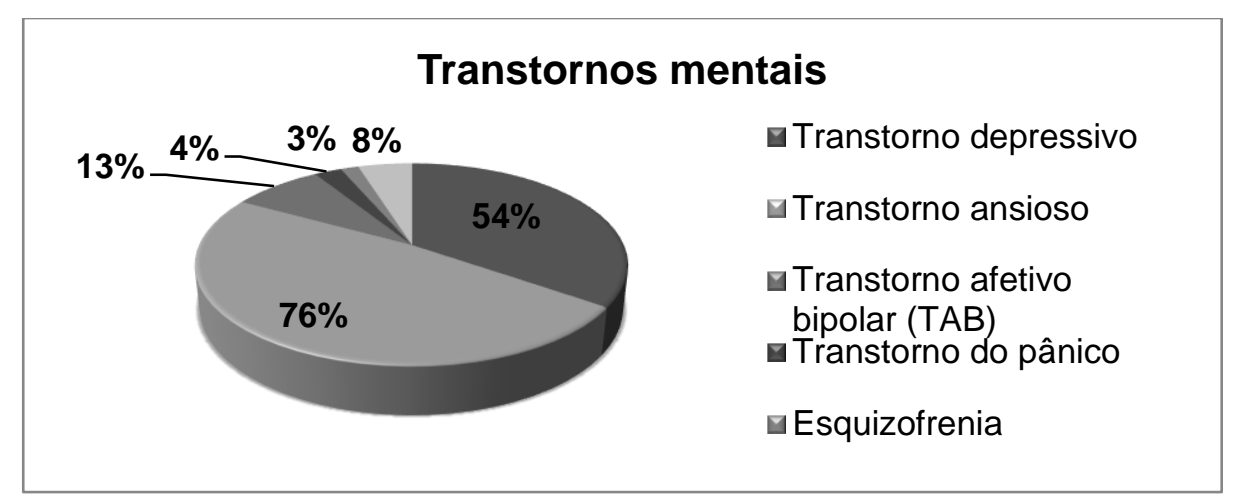

Fonte: Dados da pesquisa

\section{Perfil farmacoterapêutico}

A análise do perfil farmacoterapêutico dos pacientes vinculados ao ambulatório de cuidado interprofissional voltado para a saúde mental é de fundamental importância, 
pois ele descreve os principais medicamentos que apresentam maior aceitabilidade de acordo com os transtornos já estabelecidos e sua corroboração aos protocolos instituídos pela legislação vigente.

Foi elaborada uma tabela (Tabela 2) por meio da quantificação dos psicotrópicos que constavam prescritos nos prontuários dos pacientes e organizados por classe terapêutica. De modo que o medicamento mais prescrito se tratou de um antidepressivo pertencente à classe terapêutica dos Inibidores Seletivos da Recaptação de Serotonina (ISRS), a Sertralina, estando presente na terapia de 27 pacientes.

Outro medicamento que apresentou prevalência similar foi a Quetiapina, que se trata de um Antipsicótico de $2^{\mathrm{a}}$ geração, o qual constou também em 27 prontuários. Os Inibidores Seletivos da Recaptação de Serotonina e Noradrenalina (IRSN) também apresentaram uma quantidade relevante de prescrições, estando presente em 17 prontuários, sendo a Desvenlafaxina o medicamento mais prescrito dessa classe terapêutica.

Embora o transtorno de ansiedade seja o diagnóstico mais frequente entre os pacientes da amostra, os benzodiazepínicos foram utilizados em apenas $12 \%$ dos prontuários analisados, que equivale a 10 pacientes em uso dessa classe de medicamento, sendo o Clonazepam compreendendo a $50 \%$ das prescrições que contem essa classe terapêutica (Tabela 2).

Tabela 2 - Descrição de psicotrópicos presentes nos prontuários analisados de acordo com classe terapêutica e subsequente quantitativo por prontuário

\begin{tabular}{cccc} 
Classe terapêutica & Variáveis & N & \% \\
\hline Inibidores Seletivos & Sertalina & 27 & $61 \%$ \\
da Recaptação de & Escitalopram & 14 & $32 \%$ \\
Serotonina (ISRS) & Citalopram & 01 & $2 \%$ \\
& Fluoxetina & 01 & $2 \%$ \\
& Paroxetina & 01 & $2 \%$ \\
Inibidores Seletivos da & Total & $\mathbf{4 4}$ & \\
Recaptação de & Desvenlafaxina & 14 & $82 \%$ \\
Serotonina $\boldsymbol{e}$ & Venlafaxina & 03 & $18 \%$ \\
Noradrenalina (IRSN) & Total & $\mathbf{1 7}$ & \\
& & & \\
& Quetiapina & 27 & $70 \%$ \\
Antipsicóticos de $\mathbf{2}^{\mathbf{a}}$ & Risperidona & 02 & $3 \%$ \\
geração & Olanzapina & 01 & \\
& Total & $\mathbf{3 0}$ & \\
\hline
\end{tabular}




\begin{tabular}{cccc}
\hline & Acido Valpróico & 06 & $43 \%$ \\
Estabilizante de & Lítio & 03 & $21 \%$ \\
humor & Topiramato & 03 & $21 \%$ \\
& Carbamazepina & 01 & $7 \%$ \\
& Lamotrigina & 01 & $7 \%$ \\
Ansiolíticos - & Total & $\mathbf{1 4}$ & $50 \%$ \\
Benzodiazepínico & Clonazepam & 05 & $30 \%$ \\
& Alprazolam & 03 & $10 \%$ \\
& Bromazepam & 01 & $10 \%$ \\
Fármacos & Diazepam & 01 & $80 \%$ \\
coadjuvantes & Total & $\mathbf{1 0}$ & $10 \%$ \\
& Zolpidem & 08 & $10 \%$ \\
Antidepressivo & Betabloqueador & 01 & \\
Tricíclico & Gabapetina & 01 & $100 \%$ \\
Antipsicóticos de $\mathbf{1}^{\mathbf{a}}$ & Total & $\mathbf{1 0}$ & $50 \%$ \\
geração & Levitriptilina & 09 & $50 \%$ \\
& Total & $\mathbf{0 9}$ & \\
Inibidores da & Haloperidol & 01 & $100 \%$ \\
Recaptação de & Total & $\mathbf{0 2}$ & \\
Noradrenalina e & Bupropiona & 02 & \\
Dopamina & Total & $\mathbf{0 2}$ & \\
Antidepressivo & & 01 & \\
Tetracíclico & Mirtazapina & $\mathbf{0 1}$ & \\
& Total & & \\
\hline
\end{tabular}

Fonte: Dados da pesquisa

A tabela 2 proporciona uma descrição total acerca do uso de medicamentos presentes nos prontuários dos pacientes envolvidos na análise que são voltados para o manejo dos transtornos mentais, no entanto cabe registrar os medicamentos que se enquadram como coadjuvantes que tem como finalidade tratar sintomas adjacentes aos transtornos.

Das prescrições dos pacientes assistidos, dez continham fármacos coadjuvantes, sendo eles o Zolpidem $(n=8)$ que pertence a classe dos moduladores do sono, Betabloqueador $(n=1)$ e Gabapentina que se trata de um anticonvulsivante $(n=1)$.

\section{Metodologias utilizadas no manejo dos pacientes}

Com o advento da pandemia e a necessidade de tomada de medidas de proteção que objetivassem tanto a segurança dos pacientes como as dos profissionais da saúde, 
no ambulatório de Cuidado Farmacêutico onde ocorriam consultas interprofissionais voltadas para saúde mental de forma presencial, houve uma adaptação do serviço para dar continuidade ao atendimento durante o período da pandemia causada pela COVID19, sendo o teleatendimento a metodologia utilizada para que o serviço prosseguisse durante o período de pandemia.

O Fluxograma 1 resume a sequência de etapas da elaboração das teleconsultas e/ou telemonitoramentos que foram realizadas desde o momento inicial da pandemia da Covid-19 no Ambulatório de Cuidado Farmacêutico, tendo como intuito proporcionar uma visão geral bem como uma melhor compreensão do funcionamento do cuidado interprofissional voltado a saúde mental de modo remoto.

Fluxograma 1 - Sequência de atendimento remoto em saúde mental no Ambulatório de Cuidado Farmacêutico da UFPB durante a pandemia da COVID-19

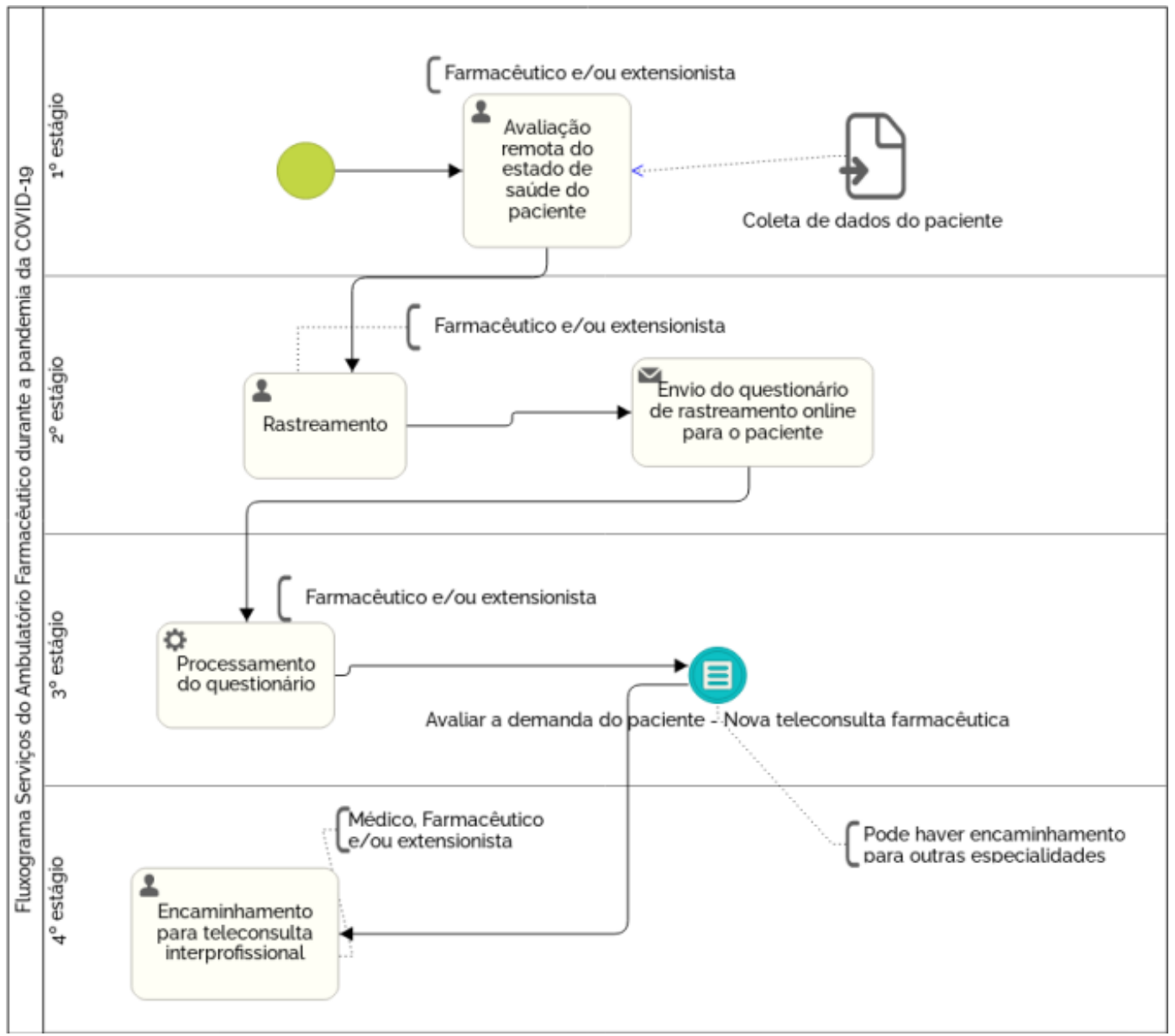

Fonte: GUIA et al., 2021

Como demonstrado no Gráfico 2, 33\% dos teleatendimentos foram teleconsultas e $93 \%$ foram telemonitoramentos. 
Grafico 2 - Distinção dos teleatendimentos realizados

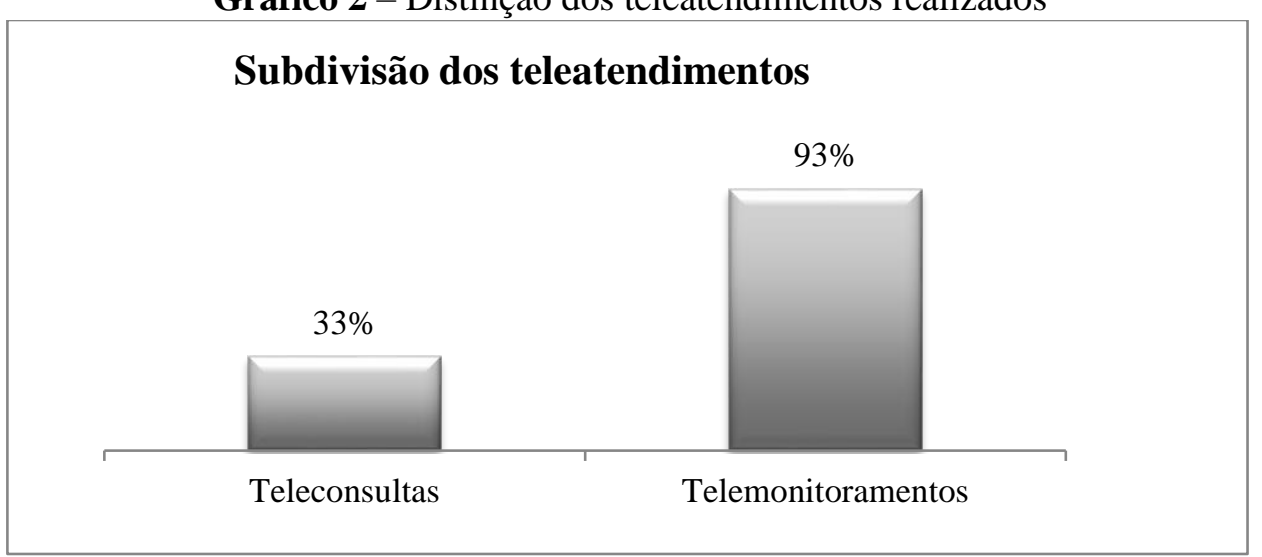

Fonte: Dados da pesquisa

Sendo o telemonitoramento posterior à realização de uma teleconsulta e era feito pelas farmacêuticas e/ou extensionistas e somente quando necessário havia a realização de uma nova teleconsulta interprofissional ou um encaminhamento para outra especialidade médica.

Cabe destacar que o mesmo paciente realizava mais de um telemonitoramento, e levando em consideração os resultados expostos, a procura pela assistência farmacêutica sobressaiu a demanda por teleconsultas interprofissionais.

\section{Avaliação e quantificação de intervenções realizadas durante os teleatendimentos}

Em relação aos telemonitoramentos, eles ocorrem unicamente com a aplicações das atribuições farmacêuticas clínicas, nos quais as Farmacêuticas e/ou extensionistas realizam uma avaliação da condição de saúde do paciente e podem sugerir intervenções junto ao médico prescritor e retorno ao paciente, favorecendo a não sobrecarga do prescritor bem como beneficiando a tomada de decisão.

Tendo isso em vista, no que concerne aos tipos de intervenções realizadas através dos teleatendimentos, a principal demanda que houve nesse período inicial da pandemia foi a de sugestão do profissional farmacêutico para a renovação de prescrições médicas (68\%). O gráfico 3 apresenta as intervenções realizadas durante os teleatendimentos. 
Grafico 3 - Intervenções realizadas durante teleatendimentos

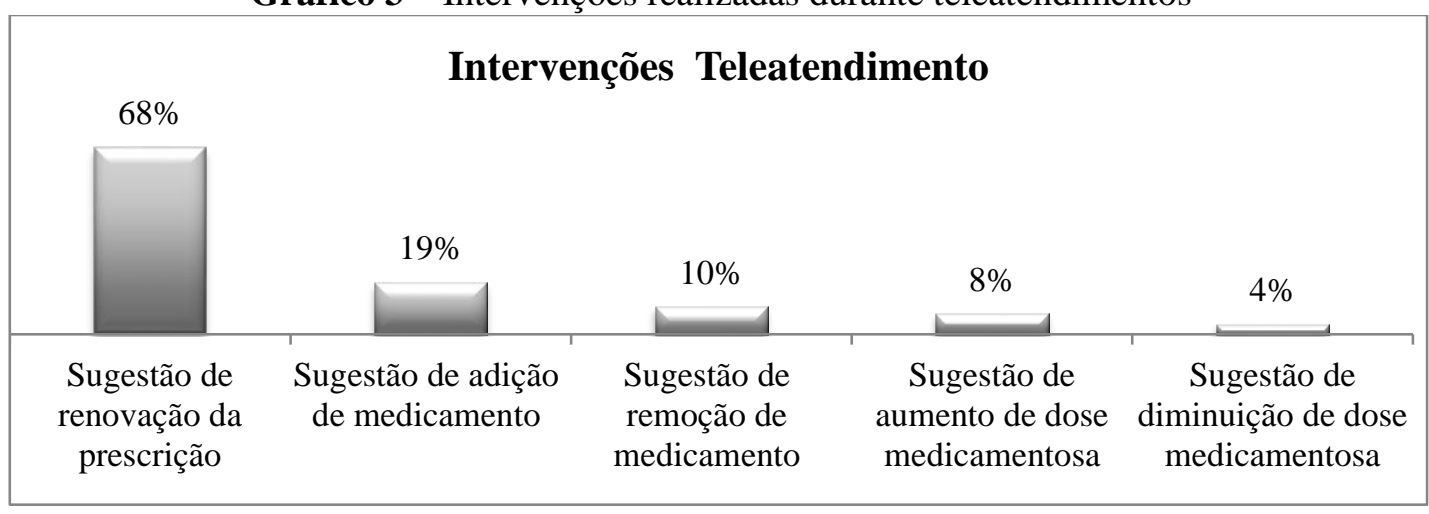

Fonte: Dados da pesquisa

Vale ressaltar que o mesmo paciente pode receber mais de uma intervenção, devido ao telemonitoramento ser constante de acordo com a necessidade do mesmo. Além disso, outras intervenções realizadas foram sugestão de substituição de classe de medicamentos, sugestão de desmame de benzodiazepínico, sugestão para alteração de horário posológico, encaminhamento para outra especialidade, entre outras.

\section{DISCUSSÃO}

O presente trabalho buscou descrever o perfil dos indivíduos vinculados ao ambulatório de cuidado farmacêutico da UFPB, os quais demandavam serviços voltados a saúde mental e sobretudo as metodologias adotadas pelos profissionais frente às adaptações necessárias no início da pandemia causada pela COVID-19. Além disso, foi realizada uma análise das intervenções realizadas para que o manejo fosse possível, assim como da farmacoterapia dos pacientes.

No que diz respeito a maior prevalência do sexo feminino (66\%) entre os dados obtidos dos prontuários, existem estudos que reforçam o predomínio do acometimento da maioria dos transtornos mentais entre as mulheres. Isso pode se dar devido a esse gênero possuir maior periodicidade em cuidados com a saúde ou ainda por uma questão de variabilidade genética (ANGST et al. 2016; CLAYTON, 2018; SAMULOWITZ et al. 2018).

Ainda há teorias que defendam que essa maior propensão se deva a sobrecarga feminina diante do cotidiano na conciliação do trabalho doméstico, matrimônio, maternidade e, sobretudo, ao fator hormonal. Estudos recentes também referenciam o 
aumento da violência doméstica durante a pandemia da COVID-19, fato esse que pode favorecer ainda mais essa estatística (OLIVEIRA, 2020; SILVA et al. 2018).

Em relação a faixa etária dos pacientes, os adultos jovens de 18 a 29 anos apresentou alta prevalência (43\%) nos registros avaliados. Por se tratar de um ambiente universitário, é natural que esse público predomine nos serviços prestados em saúde mental e em muitos outros ofertados pela própria universidade, visto que essa faixa etária corresponda a maioria da população de discentes e o âmbito em questão ocasiona nos mesmos um alto nível de estresse. Estresse esse que por sua vez é responsável por desencadear diversas complicações de saúde, dentre elas o adoecimento mental. Além disso, vale ressaltar que os adultos jovens são o público-alvo dos conteúdos educativos sobre saúde divulgados na comunidade acadêmica (LAMEU; SALAZAR; SOUZA, 2015; NINAHUAMAN et al. 2020; PEREIRA; BOTTI, 2017).

Associado a isso, segundo Elhai et al. (2017) a assiduidade dos jovens adultos em redes sociais possui diversos pontos negativos, dentre eles o desenvolvimento de transtornos mentais como os transtornos ansiosos e de depressão. Essa informação preocupa devido a maior ociosidade devido ao isolamento social, no qual essa prática tendeu a aumentar.

Auerbach et al. (2016) fez uma análise a respeito do desenvolvimento de transtornos mentais pré-existentes em universitários quando ingressavam no ensino superior e sofriam agravo ao longo da vida acadêmica, ressaltando ainda a necessidade de uma intervenção eficaz na área de saúde mental na esfera acadêmica devido o ambiente ser considerado hostil.

Dito isto, os resultados obtidos nesse trabalho corroboram os dados da literatura, os quais relacionam os elevados níveis de estresse aos quais os adultos jovens são submetidos associando-o ao desencadeamento de quadros depressivos e transtornos de ansiedade. Inclusive diversos estudos apontam o crescimento alarmante destes desde a instalação da pandemia e espera-se que haja um aumento significativo em decorrência dos efeitos em longo prazo da COVID-19 (PINTO et al. 2015;MAIA; DIAS 2020).

Ainda em relação aos achados associados à alta prevalência da faixa etária de jovens atendidos no núcleo de saúde mental durante o período inicial da pandemia, é interessante ressaltar que existiu atendimento extra-acadêmico.

Nesse sentido, o fato de os jovens adultos corresponderem ao público que apresentou maior demanda pode ter se dado por indicação de parentes ou por 
divulgações em redes sociais. Sendo esse último condizente com estudos os quais afirmam que os jovens adultos correspondem à faixa etária mais assídua nas redes sociais (MOROMIZATO et al. 2017).

Embora $76 \%$ dos prontuários investigados possuam o diagnóstico de transtorno de ansiedade, é bastante comum que ele coexista com o transtorno depressivo. De tal modo que essa coexistência, denominada como depressão ansiosa por alguns autores, dificulta o rastreio e em algumas situações traz complicações no manejo dessa comorbidade quando comparado ao manejo individual destas (COPLAN 2015; GASPERSZ et al. 2018).

Quanto à presença de outras comorbidades nos portadores de transtornos mentais, é bem sabida a coexistência do adoecimento mental em inúmeras doenças de caráter crônico. O presente estudo corrobora os anteriores que ressaltam a alta prevalência da HAS e DM com quadros de depressão e ansiedade(SOUZA et al. 2018; KHAN et al. 2019).

Além da mudança de hábitos de vida, um dos fatores que causam maior aflição nos pacientes com doenças crônicas é a necessidade contínua de cuidados médicos, muitas vezes de forma intensiva nas quais precisa de contínuas internações hospitalares interferindo assim na produtividade e gerando gastos abundantes (LIMA et al. 2020; YOONG et al. 2017).

No que tange a farmacoterapia no tratamento dos transtornos mentais, dentre eles os transtornos ansiosos e os depressivos, a utilização de medicamentos que agem em nível do sistema nervoso central (SNC) são os que possuem maior especificidade e são denominados como psicotrópicos ou psicofármacos. Sendo esses fármacos utilizados para auxiliar na reinserção social do indivíduo, ressaltando sempre a necessidade de outros métodos terapêuticos para garantia de um reestabelecimento duradouro e eficaz de sua qualidade de vida (XAVIER et al. 2014).

Dentro da classe dos medicamentos designados como psicofármacos, existem categorias que direcionam os fármacos de acordo com a dinâmica da substância no SNC. De forma abrangente, os psicofármacos implicados nos transtornos mentais são classificados como Antidepressivos, Ansiolíticos, Antipsicóticos, Estabilizadores do humor e os sedativo-hipnóticos (BRUNTON; HILAL-DANDAN, KNOLLMAN, 2019). 
Apesar de pertencerem ao grupo dos antidepressivos, os ISRS também são aplicados à farmacoterapia de outros transtornos, como nos casos de transtorno de ansiedade, visto seu potencial de neuromodulação ou neuroplasticidade. Sendo este considerado uma boa opção no que diz respeito à diminuição de recidivas e baixas taxas de abstinência. A maior prevalência do uso de antidepressivos nos prontuários analisados coincide com dados literários que investigam o perfil farmacoterapêutico de usuários da saúde mental (FULONE;LOPES, 2017; WILLIAMS et al. 2017).

Diante disso, os medicamentos da classe dos ISRS são considerados primeira linha de tratamento para transtornos de ansiedade. De maneira que a Sertralina foi o medicamento mais prescrito nos prontuários aqui analisados, correspondendo a $61 \%$ dos ISRS receitados, corroborando assim os estudos que indicavam a Sertralina como ISRS mais utilizado. Sendo essa prevalência relacionada a vantagens farmacocinéticas como tempo de meia-vida mais curta e menor probabilidade de ocasionar interações medicamentosas (SCHENKEL; COLET, 2016).

Já em relação aos ISRN, o medicamento que apresentou maior presença nos prontuários analisados foi a Desvenlafaxina, esse fato pode estar associado ao achado que o transtorno depressivo foi um dos mais prevalentes entre a população de estudo. Isso levando em consideração estudo realizado anteriormente, no qual afirma que esse medicamento apresenta bons resultados na resposta terapêutica de pacientes que possuem diagnóstico de transtorno depressivo maior (TDM). Além disso, há evidências científicas de que a Desvenlafaxina apresenta baixos níveis de interação medicamentosa, sendo considerada uma boa opção para pacientes polimedicados (CARRASCO et al. 2016; LOW; SETIA; LIMA, 2018).

Quanto ao uso de antipsicóticos, essa classe apresentou um percentual notável de uso entre os prontuários analisados, sendo a Quetiapina, um Antipsicótico de $2^{\mathrm{a}}$ geração, presente em $33 \%$ destes. Isso pode estar relacionado com a presença de pacientes que possuem diagnóstico para TAB (13\%), pois de acordo com Muneer (2015) a quetiapina apresenta eficácia terapêutica em episódios depressivos, de mania e mistos.

Para além disso, estudos recentes tem indicado o uso crescente da quetiapina em pacientes com quadro de transtorno ansioso e depressivo, fato que corrobora o fato desses serem os tipos de transtornos mais frequentes bem como o medicamento da classe mais prescrito entre os prontuários estudados. Além disso, em baixa dose a 
quetiapina apresenta potencial hipnótico importante e ajuda a poupar o uso de benzodiazepínicos (Duncan et al. 2016).

Ainda relacionado à farmacoterapia do TAB, a classe dos medicamentos dos estabilizantes de humor é considerada como primeira linha desse tipo de transtorno, sendo comumente o lítio e/ou ácido valpróico as drogas de escolha. Contudo, o Lítio passou a ter seu uso diminuído devido a sua estreita janela terapêutica. Com isso, a prevalência do ácido valpróico em relação ao lítio nos prontuários analisados pode estar relacionado com o potencial intoxicante do lítio(ELOLA et al. 2015; SANTANA; COMARELLA 2015).

Embora os psicotrópicos sejam amplamente prescritos, o manejo em saúde mental é algo bastante particular, de tal modo que é vasta a diversidade dos tratamentos mesmo entre pacientes com diagnósticos semelhantes. Isso se dá devido as particularidades de cada paciente diante dos tratamentos propostos, pois além da colaboração expressa pela autonomia do indivíduo sobre a farmacoterapia, os psicofármacos apresentam uma alta prevalência no que diz respeito a reações adversas a medicamentos (RAM)( SOUZA; KOPITTKE, 2016).

Esse fato pode ser observado na utilização da classe dos "ansiolíticos" como coadjuvantes em quadros de transtornos depressivos, no manejo de quadros de insônia e ansiedade, que não raras vezes são observadas em pacientes com transtorno depressivo no início do tratamento. No entanto, essa combinação é indicada apenas em curto prazo, como no caso da associação de benzodiazepínicos e antidepressivos(NALOTO et al. 2016).

Embora os benzodiazepínicos cumpram bem a sua função ansiolítica e sedativa, quando usados de forma irracional, manifestam respostas indesejáveis que são pontos negativos no momento de escolha terapêutica. Dentre as RAM dos benzodiazepínicos se destacam a tolerância, abstinência e dependência(MOURA et al. 2017; SOUSA; VEDANA; MIASSO, 2016).

Levando em consideração os pontos negativos da utilização de benzodiazepínicos, o baixo percentual de uso (12\%) entre os pacientes do ambulatório, favorece o que Domínguez et. al., (2016) alertaram sobre a importância do uso racional de benzodiazepínicos para melhorar a prescrição do usuário e consequentemente sua qualidade de vida. 
Os psicotrópicos são essenciais para os momentos mais agudos dos transtornos mentais, porém são cruciais para a manutenção da estabilidade do paciente. Contudo, não raras vezes as RAM dessa classe medicamentosa interferem de forma negativa na vida dos pacientes. Esse último fato resulta na necessidade constante de avaliação da farmacoterapia, a fim de dar suporte na adesão do paciente tal qual realizações de intervenções pertinentes à necessidade dos mesmos (MAZZAIA;SOUSA, 2017; SILVA; LIMA, 2017).

Por conseguinte, inúmeros países vêm considerando ao longo dos anos alternativas que utilizam da tecnologia para favorecer esse acompanhamento contínuo dos indivíduos portadores de condições crônicas, principalmente em ocasiões nas quais o paciente possui limitações físicas bem como em situações que a população reside em locais de difícil acesso ou em situações de caráter emergencial (KRUSE et al. 2018; XU et al. 2020).

No momento de crise sanitária que a instalação da pandemia da COVID-19 provoca, a telemedicina tem sido uma alternativa considerada eficaz e segura para manejo de condições clínicas que não necessitam de exame físico, como os casos de transtornos mentais. Sendo essa assistência na saúde mental vital principalmente para aqueles que atuam na linha de frente no combate a pandemia (LI et al. 2020; SILVA et al. 2020; XIANG et al. 2020).

A telemedicina, que consiste na prestação de serviços por meios remotos, possui como principal intuito a descentralização da assistência em saúde, de forma que ela pode ser classificada de acordo com a metodologia na qual ela é elaborada. De maneira geral, a teleconsulta e o telemonitoramento são os métodos mais utilizados no manejo clínico de pacientes (BUVIK et al. 2016; FLODGREN et al. 2015).

Em linhas gerais, as doenças com caráter crônico, nas quais possuem uma farmacoterapia em longo prazo devido à incidência de RAM, bem como problemas de adesão intencionais necessitam de assistência continuada. Com isso é de extrema importância que haja um acompanhamento interprofissional que objetive promover educação em saúde e também análise de eficácia do tratamento e evolução da condição clínica do paciente. Sendo videoconferência um método considerado propício para obtenção de melhores diagnósticos, bem como tomadas de decisão (RUSH; THASE, 2018; RUSH et al. 2018). 
As teleconsultas ocorreram de modo semelhante ao atendimento presencial, de modo que ocorre a coleta de dados, análise dos dados e a tomada de decisão que ocorre de forma interprofissional. Contudo o telemonitoramento consiste em uma central de atendimento para resolução de problemas que não demandam necessariamente de uma teleconsulta, como nos casos de manejo de problemas autolimitados, dúvidas em relação a posologias, acolhimento, entre outros no qual o farmacêutico clínico possui habilidades para solucionar.

Todos os indivíduos submetidos ao teleatendimento durante o período estabelecido da pandemia da COVID-19 tiveram serviços de saúde prestados por meio do aplicativo WhatsApp, de modo que as teleconsultas eram preferencialmente feitas por meio de chamadas de vídeo, enquanto o telemonitoramento ocorria por chamadas de vídeo, chamadas de voz e mensagens de texto.

O núcleo de atendimento já assistia paciente antes da pandemia, de forma presencial e sempre prezando a Interprofissionalidade, visto que a área da saúde mental se constitui como sendo uma área que apresenta melhores resultados quando conduzida de forma holística, analisando o indivíduo como o todo. Corroborando com Cleary et al. (2019), que traz evidências científicas sobre os benefícios do atendimento multiprofissional em pacientes da saúde mental principalmente no que diz respeito a decisões compartilhadas.

Tendo isso em vista, é fundamental que haja uma equipe especializada e acessível para as demandas de um paciente com transtorno mental, para que ele se sinta acolhido e ao mesmo tempo ativo nas tomadas de decisões no que concerne o seu tratamento e o manejo da sua condição clínica de modo geral. Além das vantagens já apresentadas, Leonardo et al. (2017) demonstra os benefícios do acompanhamento ambulatorial de pacientes da saúde mental, visto que consiste em um tipo de assistência que não abrange a demanda de forma suficiente.

Junior \& Amarante (2015) traz uma importante reflexão sobre a necessidade da descentralização, bem como a integralidade no que se refere ao cuidado voltado à saúde mental. Por se tratar de um momento caracterizado por desestabilização emocional em massa, a necessidade de um serviço interprofissional voltado à saúde mental que vem aumentando ao longo dos anos se encontra em demanda máxima nos dias atuais.

Nesse contexto, o cuidado farmacêutico, a partir das atribuições clínicas que são legalizadas ao profissional farmacêutico, visa aprimorar esse vínculo do paciente com 
sua farmacoterapia, tendo em mente que a utilização dos medicamentos corretos e de maneira adequada será indispensável para melhora da qualidade de vida do paciente (OLIVEIRA; FILIPIN; GIARDIN, 2015; BUKHSH et al. 2018).

Com o avanço da pandemia, a alta demanda dos telemonitoramentos (93\%) e teleconsultas (33\%) indica a importância da atuação do farmacêutico nesse âmbito. Segundo Silva et al. (2018) ao expor a dificuldade na compreensão do tratamento farmacológico dos pacientes da saúde mental, evidenciou a necessidade da assistência farmacêutica para melhor desfecho terapêutico dos mesmos.

Song et al. (2020) proporciona informações bastante relevantes acerca da inserção do profissional farmacêutico na equipe multiprofissional voltada ao cuidado de pacientes durante a pandemia da COVID-19. Ressaltando ainda mais a importância da Interprofissionalidade nos serviços de saúde, uma vez que principalmente em paciente do âmbito hospitalar fazem uso de uma gama de medicamentos incluindo os psicotrópicos. Sendo assim é indispensável a participação do farmacêutico na tomada de decisões, a fim de proporcionar sempre a melhor opção terapêutica tendo em vista o risco-benefício do paciente.

Diante do exposto, o presente trabalho destacou a relevância dos cuidados farmacêuticos associados ao serviço interprofissional voltado à área da saúde mental por meio da experiência vivenciada durante o período de adaptação dos serviços em saúde na fase inicial da pandemia no ambulatório de cuidado farmacêutico da UFPB.

\section{CONCLUSÃO}

Dado o exposto, quanto ao perfil epidemiológico da população estudada obtevese que era composto majoritariamente pelo sexo feminino e com faixa etária entre 18 a 29 anos. A respeito dos prontuários que continham diagnóstico de TM, notou-se que o mais prevalente foi o transtorno de ansiedade, seguido do transtorno de depressão.

No que tange a farmacoterapia, que se observou ser composta por psicotrópicos, constatou-se que o medicamento mais prescrito foi a classe dos antidepressivos pertencentes à classe terapêutica dos inibidores seletivos da recaptação de serotonina (ISRS), de maneira que a Sertralina foi o medicamento mais prevalente dessa classe.

Quanto a metodologia de escolha para adequação e consequente continuidade do serviço interprofissional no âmbito da saúde mental diante da pandemia da COVID-19, 
o teleatendimento foi a de escolha. De forma que os teleatendimentos foram subdividos em teleconsultas interprofissionais e telemonitoramentos, no entanto os telemonitoramentos em que ocorriam consultas farmacêuticas que proporcionavam avaliação de condição clínica dos pacientes predominou o quantitativo dos teleatendimentos.

Sobre as intervenções realizadas durante os teleatendimentos pode se observar que a mais efetuada foi a foi a sugestão de renovação da prescrição, seguida das sugestões de adição de medicamentos, sugestões de remoção de medicamentos e sugestões de aumento de dose medicamentosa.

Os resultados obtidos pela análise dos teleatendimentos enfatizam a relevância da atuação do farmacêutico clínico no manejo de condições crônicas que se estabelecem como problemas de saúde pública, como exemplo os transtornos mentais, visto que a base terapêutica dessa e outras situações clínicas é a terapia farmacológica. Terapia essa a qual o farmacêutico possui habilidades técnico-científicas significativas para favorecer os demais profissionais envolvidos no cuidado em saúde bem como principalmente os pacientes.

Diante disso, torna-se evidente a necessidade de uma assistência contínua e interprofissional dos pacientes da saúde mental, a fim de se obter tanto uma melhor evolução clínica como também menores casos de reincidências e desfechos negativos.

Para mais, o estudo apresenta uma proposta de fluxograma de atendimento virtual em saúde mental envolvendo farmacêuticos, extensionistas e médico, caracterizando uma ferramenta reprodutível para outros ambientes de saúde com atuação interprofissional que almejem exercer suas atividades de forma remota em saúde mental.

\section{AGRADECIMENTOS}

Este trabalho contou com apoio financeiro da Chamada $n^{\mathbf{o}}$ 03/2020 Produtividade em Pesquisa PROPESQ/PRPG/UFPB código do projeto de pesquisa no SIGAA PVG13392-2020. 


\section{REFERÊNCIAS}

AFONSO, P. The Impact of the COVID-19 Pandemic on Mental Health. Acta Médica Portuguesa, v. 33, n. 13, 2020. doi: https://doi.org/10.20344/amp.13877

AFONSO, P,; FIGUEIRA, L. Pandemia COVID-19: Quais são os Riscos para a Saúde Mental?. Revista Portuguesa de Psiquiatria e Saúde Mental, v. 6, n. 1, p. 2-3, 2020. Available from:

https://www.revistapsiquiatria.pt/index.php/sppsm/article/download/131/53

ANGST, J.; PAKSARIAN, D.; CUI, L., MERIKANGAS, K. R.; HENGARTNER, M. P.; AJDACIC-GROSS, V.; RÖSSLER, W., The epidemiology of common mental disorders from age 20 to 50: results from the prospective Zurich cohort Study.

Epidemiology and psychiatric sciences, v. 25, n. 1, p. 24-32, 2016.

doi: https://doi.org/10.1017/S204579601500027X

AUERBACH, R. P.; ALONSO, J.; AXINN, W. G.; CUIJPERS, P.; EBERT, D. D.; GREEN, J. G.; ... \& NOCK, M. K. Mental disorders among college students in the World Health Organization world mental health surveys. Psychological medicine, v. 46, n. 14, p. 2955-2970, 2016. doi: https://doi.org/10.1017/S0033291716001665

BERTAZONE, T. M. A.; DUCATTI, M.; DE CAMARGO, H. P. M. BATISTA, J. M. F.; KUSUMOTA, L.; MARQUEs, S. Multidisciplinary/Interdisciplinary Actions in the Care of Elderly with Alzheimer's Disease. Revista da Rede de Enfermagem do Nordeste , v. 17, n. 1, p. 144-153, 2016. doi: <10.15253/2175-6783.2016000100019>

BEZERRA, I. C.; MORAIS, J. B. D.; PAULA, M. L. D,; SILVA, T. M. R.; JORGE, M. S. Uso de Psicofármacos Na Atenção Psicossocial: Uma Análise à Luz Da Gestão Do Cuidado. Saúde em debate, v. 40, p. 148-161, 2016.

doi: https://doi.org/10.1590/0103-1104201611011

BRASIL. 2013. “RDC No 585/2013.” Diário Oficial da União, Brasília, DF, 25 set. 2013 - Seção 1, p.186.

Disponível em: http://www.cff.org.br/userfiles/file/resolucoes/585.pdf

BRUNTON, L. L.; HILAL-DANDAN, R.; KNOLLMAN B. C. As Bases

Farmacológicas Da Terapêutica de Goodman \& Gilman. 13. ed. - Porto Alegre: AMGH, 2019. ISBN 978-85-8055-615-5.

Disponível em: https://integrada.minhabiblioteca.com.br/\#/books/9788580556155/

BUKHSH, A.; KHAN, T. M.; LEE, S. W.; LEE, L. H.; CHAN, K. G.; GOH, B. H. Efficacy of pharmacist based diabetes educational interventions on clinical outcomes of adults with type 2 diabetes mellitus: a network meta-analysis. Frontiers in pharmacology, v. 9, p. 339, 2018. doi: https://doi.org/10.3389/fphar.2018.00339

BUVIK, A.; BUGGE, E.; KNUTSEN, G.; SMÅBREKKE, A.; WILSGAARD, T.

Quality of care for remote orthopaedic consultations using telemedicine: a randomised controlled trial. BMC health services research, v. 16, n. 1, p. 483, 2016.

doi: https://doi.org/10.1590/1414-462X201500040226 
CAMPOS JUNIOR, A.; \& AMARANTE, P. D. D. C. Estudo sobre práticas de cuidado em saúde mental na Atenção Primária: o caso de um município do interior do estado do Rio de Janeiro. Cadernos Saúde Coletiva, v. 23, n. 4, p. 425-435, 2015.

doi: https://doi.org/10.1590/1414-462X201500040226

CARRASCO, J. L.; KORNSTEIN, S. G.; MCINTYRE, R. S.; FAYYAD, R.; PRIETO, R.; SALAS, M.; ... \& BOUCHER, M. An integrated analysis of the efficacy and safety of desvenlafaxine in the treatment of major depressive disorder. International Clinical Psychopharmacology, v. 31, n. 3, p. 134-146, 2016.

doi: https://doi.org/10.1097/yic.0000000000000121

CLAYTON, J. A. Applying the new SABV (sex as a biological variable) policy to research and clinical care. Physiology \& behavior, v. 187, p. 2-5, 2018.

doi: https://doi.org/10.1016/j.physbeh.2017.08.012

CLEARY, M.; FOONG, A.; KORNHABER, R.; MCLEAN, L.; VISENTIN, D. C. Interprofessional collaborations for improved health care. Issues in mental health nursing, v. 40, n. 12, p. 1045-1048, 2019. https://doi.org/10.1080/01612840.2019.1655367

COPLAN, J. D.; AARONSON, C. J.; PANTHANGI, V.; KIM, Y. Treating comorbid anxiety and depression: Psychosocial and pharmacological approaches. World journal of psychiatry, v. 5, n. 4, p. 366, 2015. doi: <10.5498 / wjp.v5.i4.366>

DOMÍNGUEZ, V.; COLLARES, M.; ORMAECHEA, G.; TAMOSIUNAS, G. Uso racional de benzodiacepinas: hacia una mejor prescripción. Revista Uruguaya de Medicina Interna, v. 1, n. 3, p. 14-24, 2016. ISSN 2393-6797.

DUNCAN, D.; COOKE, L.; SYMONDS, C.; GARDNER, D.; PRINGSHEIM, T. Quetiapine use in adults in the community: a population-based study in Alberta, Canada. BMJ open, v. 6, n. 3, 2016. doi: http://dx.doi.org/10.1136/bmjopen-2015010861

ELHAI, J. D.; DVORAK, R. D.; LEVINE, J. C.; HALL, B. J. Problematic smartphone use: A conceptual overview and systematic review of relations with anxiety and depression psychopathology. Journal of affective disorders, v. 207, p. 251-259, 2017. doi: https://doi.org/10.1016/j.jad.2016.08.030

ELOLA, T. M.; MOTILLA, S. J.; VEGA, Z. G.; LLORENTE, S. S. La intoxicación por litio: urgencia orgánica en paciente psiquiátrico. Atalaya Médica Turolense, n. 8, p. 93-96, 2016.

Available: http://atalayamedica.comteruel.org/index.php/revista/article/view/118/125.

FLODGREN, G.; RACHAS, A.; FARMER, A. J.; INZITARI, M.; SHEPPERD, S. Interactive telemedicine: effects on professional practice and health care outcomes. Cochrane Database of Systematic Reviews, n. 9, 2015. doi: https://dx.doi.org/10.1002\%2F14651858.CD002098.pub2 
FULONE, I.; LOPES, L.C., Potentially inappropriate prescriptions for elderly people taking antidepressant: comparative tools. BMC geriatrics, v. 17, n. 1, p. 278, 2017. doi: https://doi.org/10.1186/s12877-017-0674-2

GASPERSZ, R.; NAWIJN, L.; LAMERS, F.; PENNINX, B. W. Patients with anxious depression: overview of prevalence, pathophysiology and impact on course and treatment outcome. Current opinion in psychiatry, v. 31, n. 1, p. 17-25, 2018. doi: <10.1097/YCO.0000000000000376>

HARZHEIM, E.; MARTINS, C. C.; WOLLMANN, L.; PEDEBOS, L. A.; DE ALMEIDA FALLER, L.; DAS CHAGAS MARQUES, M.; ... LEAL, M. H. Ações federais para apoio e fortalecimento local no combate ao COVID-19: a Atenção Primária à Saúde (APS) no assento do condutor. Ciência \& Saúde Coletiva, v. 25, p. 2493-2497, 2020. doi: https://doi.org/10.1590/1413-81232020256.1.11492020

KHAN, P.; QAYYUM, N.; MALIK, F.; KHAN, T.; KHAN, M.; TAHIR, A. Incidence of anxiety and depression among patients with Type 2 diabetes and the predicting factors. Cureus, v. 11, n. 3, 2019. doi: https://dx.doi.org/10.7759\%2Fcureus.4254

LAMEU, J. D. N.; SALAZAR, T. L.; SOUZA, W. F. D. Prevalence of stress symptoms among students of a public university. Psicologia da Educação, n. 42, p. 13-22, 2016. doi: https://doi.org/10.1016/j.physbeh.2017.08.012

LEONARDO, B. C.; CUNHA, D. F.; SAKAE, T. M.; REMOR, K. V. T. Prevalência de Transtornos Mentais e Utilização de Psicofármacos em Pacientes Atendidos em um Ambulatório médico de Especialidades. Arquivos Catarinenses de Medicina, v. 46, n. 2, p. 39-52, 2017.

Available: http://www.acm.org.br/acm/seer/index.php/arquivos/article/view/268

LI, W.; YANG, Y.; LIU, Z. H.; ZHAO, Y. J.; ZHANG, Q.; ZHANG, L.; ... XIANG, Y. T. Progression of mental health services during the COVID-19 outbreak in China.

International journal of biological sciences, v. 16, n. 10, p. 1732, 2020. doi: https://dx.doi.org/10.7150\%2Fijbs.45120

LIMA, C. D. A. D.; OLIVEIRA, R. C. D.; OLIVEIRA, S. A. G. D.; SILVA, M. A. S. D.; LIMA, A. D. A.; ANDRADE, M. S.; PINHO, C. M. Qualidade de vida, ansiedade e depressão em pacientes com doença pulmonar obstrutiva crônica. Revista Brasileira de Enfermagem, v. 73, 2020. doi: https://doi.org/10.1590/0034-7167-2019-0423

LOW, Y.; SETIA, S.; LIMA, G. Drug-drug interactions involving antidepressants: focus on desvenlafaxine. Neuropsychiatric disease and treatment, 14, 567-580, 2018. doi: https://doi.org/10.2147/NDT.S157708

MAIA, B. R.; DIAS, P. C. Ansiedade, depressão e estresse em estudantes universitários: o impacto da COVID-19. Estudos de Psicologia (Campinas), v. 37, 2020. doi: https://doi.org/10.1590/1982-0275202037e200067

MAZZAIA, M. C.; SOUZA, M. A. Adesão ao tratamento no Transtorno Afetivo Bipolar: percepção do usuário e do profissional de saúde. Revista Portuguesa de Enfermagem de Saúde Mental, n. 17, p. 34-42, 2017. 
doi: http://dx.doi.org/10.19131/rpesm.0181

MOROMIZATOI, M. S.; FERREIRAI, D. B. B.; DE SOUZAI, L. S. M.; LEITEI, R. F.; MACEDOI, F. N.; PIMENTELI, D. O uso de Internet e redes Sociais e a relação com Indícios de ansiedade e Depressão em Estudantes de medicina The use of the Internet and Social Networks and the relationship with Symptoms of anxiety and. Revista Brasileira de Educação Médica, v. 41, n. 4, p. 497-504, 2017. doi: http://dx.doi.org/10.1590/1981-52712015v41n4RB20160118

MOURA, D. C. N.; PINTO, J. R.; MARTINS, P.; DE ARRUDA PEDROSA, K.; CARNEIRO, M. D. G. D. Uso abusivo de psicotrópicos pela demanda da estratégia saúde da família: revisão integrativa da literatura. SANARE-Revista de Políticas Públicas, v. 15, n. 2, 2016.

Available: https://sanare.emnuvens.com.br/sanare/article/view/1048

MUNEER, A. Pharmacotherapy of bipolar disorder with quetiapine: a recent literature review and an update. Clinical Psychopharmacology and Neuroscience, v. 13, n. 1, p. 25, 2015. doi: https://dx.doi.org/10.9758\%2Fcpn.2015.13.1.25

NALOTO, D. C. C.; LOPES, F. C.; BARBERATO FILHO, S.; LOPES, L. C.; DEL FIOL, F. D. S.; BERGAMASCHI, C. D. C. Prescrição de benzodiazepínicos para adultos e idosos de um ambulatório de saúde mental. Ciência \& Saúde Coletiva, v. 21, p. 1267-1276, 2016. doi: https://doi.org/10.1590/1413-81232015214.10292015

NDIBU MUNTU KEBA KEBE, N.; CHIOCCHIO, F.; BAMVITA, J. M.; FLEURY, M. J. Profiling mental health professionals in relation to perceived interprofessional collaboration on teams. SAGE open medicine vol. 7 2050312119841467. 29 Mar. 2019, doi: <10.1177/2050312119841467>

NINAHUAMAN, S. L.; DE ANDRADE, V. C. G.; NINAHUAMAN, M. F. M. L.; DA SILVA, I. C.; MONTEIRO, M. A.; ABDALA, G. A. Estresse, transtornos mentais nãopsicóticos e expectativa de vida em alunos de cursos superiores noturnos. Life Style, v. 6, n. 2, p. 60-72, 2019. doi: http://dx.doi.org/10.19141/2237-3756.lifestyle.v6.n2.p60-7

OLIVEIRA, A. L., A espacialidade aberta e relacional do lar: a arte de conciliar maternidade, trabalho doméstico e remoto na pandemia da COVID-19. Revista Tamoios, v. 16, n. 1, 2020. doi: https://doi.org/10.12957/tamoios.2020.50448

OLIVEIRA, R. E. M.; FILIPIN, M. D. V.; GIARDINI, M. H. Intervenções farmacêuticas destinadas à otimização da adesão ao tratamento medicamentoso de um paciente. Revista Eletrônica de Farmácia, v. 12, n. 2, p. 39-51, 2015. doi: https://doi.org/10.5216/ref.v12i2.34346

Organization, World Health. 2017. "Depression and Other Common Mental Disorders: Global Health Estimates." Global Health Estimates 48(1): 56-60.

ORNELL, F. E. L. I. P. E.; SCHUCH, J. B.; SORDI, A. O.; KESSLER, F. H. P. "Pandemic fear" and COVID-19: mental health burden and strategies. Brazilian Journal of Psychiatry, v. 42, n. 3, p. 232-235, 2020. doi:http://dx.doi.org/10.1590/1516-4446-2020-0008 
PEREIRA, C. C. M.; BOTTI, N. C. L. O suicídio na comunicação das redes sociais virtuais: revisão integrativa da literatura. Revista Portuguesa de Enfermagem de Saúde Mental, n. 17, p. 17-24, 2017. doi: http://dx.doi.org/10.19131/rpesm.0179

PEREIRA, M. D.; DE OLIVEIRA, L. C.; COSTA, C. F. T.; DE OLIVEIRA BEZERRA, C. M.; PEREIRA, M. D.; DOS SANTOS, C. K. A.; DANTAS, E. H. M. A pandemia de COVID-19, o isolamento social, consequências na saúde mental e estratégias de enfrentamento: uma revisão integrativa. Research, Society and Development, v. 9, n. 7, p. e652974548-e652974548, 2020. doi: http://dx.doi.org/10.33448/rsd-v9i7.4548

PINTO, J. C.; MARTINS, P.; PINHEIRO, T. B.; OLIVEIRA, A. C. Ansiedade, depressão e stresse: um estudo com jovens adultos e adultos portugueses. Psicologia, Saúde \& Doenças, v. 16, n. 2, p. 148-163, 2015. doi: http://dx.doi.org/10.15309/15psd160202

RUSH, A. J.; THASE, M. E., Improving depression outcome by patient-centered medical management. American Journal of Psychiatry, v. 175, n. 12, p. 1187-1198, 2018. doi: https://doi.org/10.1176/appi.ajp.2018.18040398

RUSH, K. L.; HOWLETT, L.; MUNRO, A.; BURTON, L. Videoconference compared to telephone in healthcare delivery: A systematic review. International journal of medical informatics, v. 118, p. 44-53, 2018.

doi: https://doi.org/10.1016/j.ijmedinf.2018.07.007

SAMULOWITZ, A.; GREMYR, I.; ERIKSSON, E.; HENSING, G. "Brave men" and "emotional women": A theory-guided literature review on gender bias in health care and gendered norms towards patients with chronic pain. Pain Research and Management, v. 2018, 2018. doi: https://doi.org/10.1155/2018/6358624

SANTANA, J. S.; COMARELLA, L. Bipolar disorder (mania and hypomania) and pharmacological treatment. Visão Acadêmica, v. 16, n. 1, 2015.

doi: http://dx.doi.org/10.5380/acd.v16i1.40125

SCHENKEL, M.; DE FÁTIMA COLET, C. Uso de antidepressivos em um município do Rio Grande do Sul. Arquivos de Ciências da Saúde da UNIPAR, v. 20, n. 1, 2016. doi: https://doi.org/10.25110/arqsaude.v20i1.2016.5220

SCOTT KRUSE, C.; KAREM, P.; SHIFFLETT, K.; VEGI, L.; RAVI, K.; BROOKS, M. Evaluating barriers to adopting telemedicine worldwide: A systematic review. Journal of telemedicine and telecare, v. 24, n. 1, p. 4-12, 2018 doi: https://dx.doi.org/10.1177\%2F1357633X16674087

SEVERO, A. K.; DIMENSTEIN, M. Rede e intersetorialidade na atenção psicossocial: contextualizando o papel do ambulatório de saúde mental. Psicol., Ciênc. Prof. (Impr.), Brasília, DF, v. 31, n. 3, p. 640-655, 2011. doi: https://doi.org/10.1590/S141498932011000300015 
SILVA, J. K.; DE ALBUQUERQUE, S. C.; SANTOS, S. S. N.; DOS SANTOS, V. M. F.; DE FARIAS, K. F.; DE SOUZA FIGUEIREDO, E. V. M.; DOS SANTOS, A. C. M. A relação entre a infecção por coronavírus e susceptibilidade a transtornos mentais e o risco de suicídio: o que a literatura tem evidenciado?. Journal of Health \& Biological Sciences, v. 8, n. 1, p. 1-7, 2020. doi: http://dx.doi.org/10.12662/23173076jhbs.v8i1.3242.p1-7.2020

SILVA, P. A. D. S. D.; ROCHA, S. V.; SANTOS, L. B.; SANTOS, C. A. D.; AMORIM, C. R.; VILELA, A. B. A. Prevalência de transtornos mentais comuns e fatores associados entre idosos de um município do Brasil. Ciência \& saúde coletiva, v. 23, p. 639-646, 2018. doi: https://doi.org/10.1590/1413-81232018232.12852016

SILVA, S. N.; LIMA, M. G. Prescrições Em Serviços de Saúde Mental: Aspectos Legais e Indicadores Do Uso Racional de Medicamentos. Scientia Medica, v. 27, n. 3 , p. 6, 2017b. doi: <10.15448/1980-6108.2017.3.25597>

SONG, Z.; HU, Y.; ZHENG, S.; YANG, L.; ZHAO, R. Hospital pharmacists' pharmaceutical care for hospitalized patients with COVID-19: recommendations and guidance from clinical experience. Research in Social and Administrative Pharmacy, v. 17, n. 1, p. 2027-2031, 2021. https://doi.org/10.1016/j.sapharm.2020.03.027

SOUSA, L. P. D. C.; VEDANA, K. G. G.; MIASSO, A. I. Adesão ao tratamento medicamentoso por pessoas com transtorno de ansiedade. Cogitare Enfermagem, v. 21, n. 1, 2016. doi: http://dx.doi.org/10.5380/ce.v21i1.43510

SOUZA, G. N. P. D.; ALVES, R. J. R.; SOUZA, L. P. S.; ROSA, A. J. Prevalência de sintomas depressivos e/ou ansiosos em pessoas com hipertensão arterial sistêmica e/ou diabetes mellitus. Revista Portuguesa de Enfermagem de Saúde Mental, n. 20, p. 4350, 2018. doi: http://dx.doi.org/10.19131/rpesm.0225

SOUZA, M. S. F.; KOPITTKE, L. Adesão ao tratamento com psicofármacos: fatores de proteção e motivos de não adesão ao tratamento farmacológico. Revista de APS, v. 19, n. 3, 2016. Available: https://periodicos.ufjf.br/index.php/aps/article/view/15497

USHER, K.; BHULLAR, N.; JACKSON, D. Life in the pandemic: Social isolation and mental health. Journal of Clinical Nursing, 2756-57, 2020.

doi:https://doi.org/10.1111/jocn.15290

VASCONCELOS, M. G. F.; JORGE, M. S. B.; CATRIB, A. M. F.; BEZERRA, I. C.; FRANCO, T. B. Projeto terapêutico em Saúde Mental: práticas e processos nas dimensões constituintes da atenção psicossocial. Interface-Comunicação, Saúde, Educação, v. 20, p. 313-323, 2016. doi:https://doi.org/10.1590/1807-57622015.0231

VELÁSQUEZ, J. R. M. Teleconsulta en la pandemia por Coronavirus: desafíos para la telemedicina pos-COVID-19. Revista Colombiana de Gastroenterología, v. 35, n. Supl. 1, p. 5-16, 2020. doi:https://doi.org/10.22516/25007440.543

WILLIAMS, T.; HATTINGH, C. J.; KARIUKI, C. M.; TROMP, S. A.; VAN BALKOM, A. J.; IPSER, J. C.; STEIN, D. J. Pharmacotherapy for social anxiety 
disorder (SAnD). Cochrane Database of Systematic Reviews, n. 10, 2017. doi:

https://dx.doi.org/10.1002\%2F14651858.CD001206.pub3

XAVIER, M. S.; TERRA, M.G.; SILVA, C. T.; SOUTO, V. T.; MOSTRADEIRO, S. C. T. S.; VASCONCELOS, R. O. A utilização de psicofármacos em indivíduos com transtorno mental em acompanhamento ambulatorial. Enfermería Global, v. 13, n. 4, p. 114-137, 2014. doi: https://doi.org/10.6018/eglobal.13.4.201121

XIANG, Y. T.; YANG, Y.; LI, W.; ZHANG, L.; ZHANG, Q.; CHEUNG, T.; NG, C. H. Timely Mental Health Care for the 2019 Novel Coronavirus Outbreak Is Urgently Needed. The lancet psychiatry, v. 7, n. 3, p. 228-229, 2020.

http://dx.doi.org/10.1016/S2215-0366(20)30046-8.

XU, H.; HUANG, S.; QIU, C.; LIU, S.; DENG, J.; JIAO, B.; ... YAN, L. Monitoring and Management of Home-Quarantined Patients With COVID-19 Using a WeChatBased Telemedicine System: Retrospective Cohort Study. Journal of Medical Internet Research, v. 22, n. 7, p. e19514, 2020. doi: https://doi.org/10.2196/19514

YOONG, R. K.; MOOPPIL, N.; KHOO, E. Y.; NEWMAN, S. P.; LEE, V. Y.; KANG, A. W.; GRIVA, K. Prevalence and determinants of anxiety and depression in end stage renal disease (ESRD). A comparison between ESRD patients with and without coexisting diabetes mellitus. Journal of psychosomatic research, v. 94, p. 68-72, 2017. doi: https://doi.org/10.1016/j.jpsychores.2017.01.009

ZANELLA, M.; LUZ, H. H. V.; BENETTI, I. C.; ROBERTI JUNIOR, J. P. Medicalização e saúde mental: Estratégias alternativas. Revista Portuguesa de Enfermagem de Saúde Mental, n. 15, p. 53-62, 2016.

doi: http://dx.doi.org/10.19131/rpesm.0132 